\title{
THE SECOND PART OF A MEMOIR ON THE DEVELOPMENT OF THE DISTURBING FUNCTION IN THE LUNAR AND PLANETARY THEORIES.
}

[From the Memoirs of the Royal Astronomical Society, vol. xxxix. (1872), pp. 55-74. Read January 12, 1872.]

THE present communication is a sequel to my paper, "The First Part of a Memoir on the Development of the Disturbing Function in the Lunar and Planetary Theories," Memoirs R.A.S., vol. xxviII. (1859), pp. 187-215, [214], and I have therefore entitled it as above, but it, in fact, relates only to the Planetary Theory. In the First Part, I gave in effect, but not explicitly, an expression for the general coefficient $D\left(j, j^{\prime}\right)$ in terms of the coefficients of the multiple cosines of $\theta$ in the expansions of the several powers $\left(r^{2}+r^{\prime 2}-2 r r^{\prime} \cos \theta\right)^{-s-\frac{1}{2}}$, or say $\left(a^{2}+a^{\prime 2}-2 a a^{\prime} \cos \theta\right)^{-8-\frac{1}{2}}$; viz., at the foot of page 208 I speak of the term involving $\cos \left(j U+j^{\prime} U^{\prime}\right)$ as having a certain given value; the term in question is $D\left(j, j^{\prime}\right) \cos \left(j U+j^{\prime} U^{\prime}\right)$; and consequently the expression for $D\left(j, j^{\prime}\right)$ is

$$
D\left(j, j^{\prime}\right)=\Sigma \frac{\Pi_{1}\left(x-\frac{1}{2}\right)}{\Pi x} \eta^{2 x} \Sigma M_{x}{ }^{2} R_{x}{ }^{2}
$$

the omission was, however, a material one, inasmuch as this expression for the general coefficient serves to connect my formulæ with Leverrier's development, Annales de l'Observ. de Paris, t. I. (1855), pp. $275-330$ and $358-383$, and I resume the question for the purpose of applying it.

Formula for the general Coefficient $D\left(j, j^{\prime}\right)$.

In the First Part, the reciprocal of the distance of the two planets, or function

$$
\left\{r^{2}+r^{\prime 2}-2 r r^{\prime}\left(\cos U \cos U^{\prime}+\sin U \sin U^{\prime} \cos \Phi\right)\right\}^{-\frac{1}{2}}
$$


is taken to be developed in multiple cosines of $U, U^{\prime}$, the general term being

$$
D\left(j, j^{\prime}\right) \cos \left(j U+j^{\prime} U^{\prime}\right),
$$

where $j, j^{\prime}$ have each of them any integer value from $-\infty$ to $+\infty$ (zero not excluded), but so that $j, j^{\prime}$ are simultaneously even or simultaneously odd. We have $D\left(-j,-j^{\prime}\right)=D\left(j, j^{\prime}\right)$ and $D\left(j^{\prime}, j\right)=D\left(j, j^{\prime}\right)$; and it hence appears that the really distinct values of the coefficient may be taken to be those for which $j$ is not negative, and as regards absolute magnitude is not less than $j^{\prime}$; and for such values of $j$, $j^{\prime}$ we have the abovementioned expression

$$
D\left(j, j^{\prime}\right)=\Sigma \frac{\Pi_{1}\left(x-\frac{1}{2}\right)}{\Pi x} \eta^{2 x} \Sigma M_{x}{ }^{2} R_{x}{ }^{2}
$$

which I proceed to explain and develope.

$\Pi_{1}\left(x-\frac{1}{2}\right)$ and $\Pi x$ (x being a positive integer) denote respectively $\frac{1}{2} \cdot \frac{3}{2} \ldots\left(x-\frac{1}{2}\right)$, and $1.2 .3 \ldots x$; in particular for $x=0$, the value of each factorial is $=1$.

$\eta$ denotes $\sin \frac{1}{2} \Phi$.

The coefficients $R_{x}{ }^{3}$ are those of the multiple cosines in certain developments, viz. we have

$$
r^{x} r^{\prime x}\left\{r^{2}+r^{\prime 2}-2 r r^{\prime} \cos \left(U-U^{\prime}\right)\right\}^{-x-\frac{1}{2}}=\Sigma R_{x}^{i} \cos i\left(U-U^{\prime}\right),
$$

where, as usual, $i$ extends from $-\infty$ to $\infty$ and $R_{x}{ }^{-i}=R_{x}{ }^{i}$. Writing with Leverrier

$$
\begin{array}{r}
\left(a^{2}+a^{\prime 2}-2 a a^{\prime} \cos H\right)^{-\frac{1}{2}}=\frac{1}{2} \Sigma A^{i} \cos i H, \\
a a^{\prime}\left(a^{2}+a^{\prime 2}-2 a a^{\prime} \cos H\right)^{-\frac{3}{2}}=\frac{1}{2} \Sigma B^{i} \cos i H, \\
a^{2} a^{\prime 2}\left(a^{2}+a^{\prime 2}-2 a a^{\prime} \cos H\right)^{-\frac{5}{2}}=\frac{1}{2} \Sigma C^{i} \cos i H, \\
a^{3} a^{\prime 3}\left(a^{2}+a^{\prime 2}-2 a a^{\prime} \cos H\right)^{-\frac{7}{2}}=\frac{1}{2} \Sigma D^{i} \cos i H,
\end{array}
$$

then $2 R_{0}^{i}, 2 R_{1}^{i}, 2 R_{2}^{i}, 2 R_{3}^{i}$ are the same functions of $r, r^{\prime}$ that $A^{i}, B^{i}, C^{i}, D^{i}$ respectively are of $a, a^{\prime}$.

The expression of $M_{x}{ }^{2}$ is

$$
M_{x}{ }^{2}=(-)^{x-\frac{1}{2}\left(j+j^{\prime}\right)} \frac{\Pi x}{\Pi \frac{1}{2}(x-j-9) \Pi \frac{1}{2}\left(x+j^{\prime}+9\right)} \frac{\Pi x}{\Pi \frac{1}{2}(x-j+9) \Pi \frac{1}{2}\left(x+j^{\prime}-9\right)} ;
$$

and, finally, in the expression for $D\left(j, j^{\prime}\right), x$ has every integer value from 0 to $\infty$, and, for any given value of $x, 9$ extends by steps of two units from the inferior value $-\left(x-j^{\prime}\right)$ to the superior value $x-j$.

It is convenient to write $x=\frac{1}{2}\left(j+j^{\prime}\right)+s$; we have then 9 extending from $-\frac{1}{2}\left(j-j^{\prime}\right)-s$ to $-\frac{1}{2}\left(j-j^{\prime}\right)+s$, or writing $9=-\frac{1}{2}\left(j-j^{\prime}\right)+\theta, \theta$ has the $s+1$ values $s$, $s-2, s-4, \ldots-s$, viz. for $s=2 p+1$ the values are $\pm 1, \pm 3, \ldots \pm(2 p+1)$, and for $s=2 p$ they are $0, \pm 2, \pm 4, \ldots \pm 2 p$. 
Making these changes we have

where

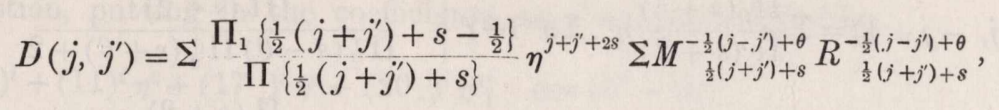

$$
M_{\frac{1}{2}\left(j+j^{\prime}\right)+s}^{-\frac{1}{2}\left(j-j^{\prime}\right)+\theta}=(-)^{s} \frac{\Pi\left\{\frac{1}{2}\left(j+j^{\prime}\right)+s\right\}}{\Pi \frac{1}{2}(s-\theta) \Pi \frac{1}{2}\left(j+j^{\prime}+s+\theta\right)} \Pi \frac{\Pi\left\{\frac{1}{2}\left(j+j^{\prime}\right)+s\right\}}{\frac{1}{2}(s+\theta) \Pi \frac{1}{2}\left(j+j^{\prime}+s-\theta\right)},
$$

viz. this is $(-)^{s}$ into the product of two binomial coefficients, each belonging to the exponent $\frac{1}{2}\left(j+j^{\prime}\right)+s$.

Particular Cases, $j+j^{\prime}=0,2,4,6$, being those required in the Planetary Theory.

Considering successively the cases $j+j^{\prime}=0,2,4,6$, we have, first,

$$
D(j,-j)=\Sigma \frac{\Pi_{1}\left(s-\frac{1}{2}\right)}{\Pi s} \eta^{2 s} \Sigma(-)^{s}\left\{\frac{\Pi s}{\Pi_{\frac{1}{2}(s-\theta) \Pi \frac{1}{2}(s+\theta)}}\right\}^{2} R_{s}^{-j+\theta}
$$

which, developed as far as $\eta^{6}$, is

$$
\begin{aligned}
D(j,-j)= & \frac{1}{2} A^{-j} \\
& -\frac{1}{2} \eta^{2} \frac{1}{2}\left(B^{-j+1}+B^{-j-1}\right) \\
& +\frac{1.3}{2.4} \eta^{4} \frac{1}{2}\left(C^{-j+2}+4 C^{-j}+C^{-j-2}\right) \\
& -\frac{1.3 \cdot 5}{2.4 \cdot 6} \eta^{6 \frac{1}{2}}\left(D^{-j+3}+9 D^{-j+1}+9 D^{-j-1}+D^{-j-3}\right)
\end{aligned}
$$

where, and in what immediately follows, $A, B, C, D$ are used to denote functions (not of $\left(a, a^{\prime}\right)$, but $)$ of $r, r^{\prime}$.

Secondly,

$$
D(j,-j+2)=\Sigma \frac{\Pi_{1}\left(s+\frac{1}{2}\right)}{\Pi(s+1)} \eta^{2} \Sigma \eta^{2 s}\left\{(-)^{s} \frac{\Pi(s+1)}{\Pi_{\frac{1}{2}(s-\theta) \Pi \frac{1}{2}(s+\theta)+1}}\right.
$$

which, developed to $\eta^{6}$, is

$$
\left.\times \frac{\Pi(s+1)}{\Pi \frac{1}{2}(s+\theta) \Pi \frac{1}{2}(s-\theta)+1} R_{s+1}^{-j+1+\theta}\right\},
$$

$$
\begin{aligned}
& D(j,-j+2)=\eta^{2}\left\{\quad \frac{1}{2} \quad \cdot \frac{1}{2} B^{-j+1}\right. \\
& -\frac{1.3}{2.4} \eta^{2} \cdot \frac{1}{2}\left(2 C^{-j+2}+2 C^{-j}\right), \\
& \left.+\frac{1.3 .5}{2.4 .6} \eta^{4} \cdot \frac{1}{2}\left(3 D^{-j+3}+9 D^{-j+1}+3 D^{-j-1}\right)\right\} .
\end{aligned}
$$

C. VII. 
Thirdly,

$$
\begin{aligned}
D(j,-j+4)=\Sigma \frac{\Pi_{1}\left(s+\frac{3}{2}\right)}{\Pi(s+2)} \eta^{4} \cdot \Sigma \eta^{2 s}(-)^{s}\{ & \frac{\Pi(s+2)}{\Pi_{\frac{1}{2}}(s-\theta) \Pi \frac{1}{2}(s+\theta)+2} \\
& \left.\times \frac{\Pi(s+2)}{\Pi_{\frac{1}{2}}(s+\theta) \Pi_{\frac{1}{2}}(s-\theta)+2} R_{s+2}^{-j+2 \theta}\right\},
\end{aligned}
$$

which, developed to $\eta^{6}$, is

$$
\begin{aligned}
D(j,-j+4)=\eta^{4}\{ & \frac{1.3}{2.4} \cdot \frac{1}{2} C^{-j+2} \\
& \left.-\frac{1.3 .5}{2.4 .6} \eta^{2} \cdot \frac{1}{2}\left(3 D^{-j+3}+3 D^{-j+1}\right)\right\}:
\end{aligned}
$$

and, fourthly,

$$
\begin{aligned}
& D(j,-j+6)=\Sigma \frac{\Pi_{1}\left(s+\frac{5}{2}\right)}{\Pi(s+3)} \eta^{6} \Sigma \eta^{2 s}(-)^{s}\left\{\begin{array}{l}
\Pi \frac{\Pi(s+3)}{\Pi \frac{1}{2}(s-\theta) \Pi \frac{1}{2}(s+\theta)+3}
\end{array}\right. \\
& \left.\times \frac{\Pi(s+3)}{\Pi \frac{1}{2}(s+\theta) \Pi \frac{1}{2}(s-\theta)+3} R_{s+3}^{-j+3+\theta}\right\},
\end{aligned}
$$

which, developed to $\eta^{6}$, is simply

$$
D(j,-j+6)=\eta^{6} \frac{1 \cdot 3 \cdot 5}{2 \cdot 4 \cdot 6} \cdot \frac{1}{2} D^{-j+3} .
$$

The foregoing formulæ, although obtained on the supposition $j=0$, or positive, apply without alteration to the case $j$-negative, and the entire series of terms of an order not exceeding 6 as regards $\eta$ may be written,

$$
\begin{array}{cl}
D(j,-j) & \cos \left(j U-j U^{\prime}\right) \\
+2 D(j,-j+2) & \cos \left(j U+(-j+2) U^{\prime}\right) \\
+2 D(j,-j+4) & \cos \left(j U+(-j+4) U^{\prime}\right) \\
+2 D(j,-j+6) & \cos \left(j U+(-j+6) U^{\prime}\right),
\end{array}
$$

where $j$ has every integer value from $-\infty$ to $+\infty$.

\section{COmparison with LEVERRIER.}

This is in fact what Leverrier's expression becomes on putting therein $e=e^{\prime}=0$. To verify this, observe that Leverrier having defined his $A^{i}, B^{i}, C^{i}, D^{i}$, as above, writes further

$$
\begin{aligned}
& E^{i}=\frac{1}{2}\left(B^{i-1}+B^{i+1}\right) \\
& G^{i}=\frac{3}{8}\left(C^{i-2}+4 C^{i}+C^{i+2}\right) \\
& H^{i}=\frac{5}{16}\left(D^{i-3}+9 D^{i+1}+9 D^{i+1}+D^{i+3}\right) \\
& L^{i}=\frac{3}{4}\left(C^{i-2}+C^{i}\right) \\
& S^{i}=\frac{15}{16}\left(D^{i-3}+3 D^{i-1}+D^{i+1}\right) \\
& T^{i}=\frac{15}{16}\left(D^{i-3}+D^{i-1}\right)
\end{aligned}
$$


(consequently $E^{-i}=E^{i}, G^{-i}=G^{i}, H^{-i}=H^{i}, L^{-i+2}=L^{i}, S^{-i+2}=S^{i}, T^{-i+4}=T^{i}$ ), and that the terms in question, putting in the coefficients $e=e^{\prime}=0$, are with him

$$
\begin{aligned}
\left\{(1)^{i}+(11)^{i} \eta^{2}+(17)^{i} \eta^{4}+(20)^{i} \eta^{6}\right\} & \cos \left(i l^{\prime}-i \lambda\right), \\
\left\{(212)^{i} \eta^{2}+(218)^{i} \eta^{4}+(221)^{i} \eta^{6}\right\} & \cos \left[i l^{\prime}-(i-2) \lambda-2 \tau^{\prime}\right], \\
\left\{(372)^{i} \eta^{4}+(375)^{i} \eta^{6}\right\} & \cos \left[i l^{\prime}-(i-4) \lambda-4 \tau^{\prime}\right] \\
\left\{(449)^{i} \eta^{6}\right\} & \cos \left[i l^{\prime}-(i-6) \lambda-6 \tau^{\prime}\right],
\end{aligned}
$$

where, substituting for $(1)^{i},(11)^{i}$, \&c., their values, the coefficients are

$$
\begin{aligned}
& \frac{1}{2} A^{i}-\eta^{2} \frac{1}{2} E^{i}+\eta^{4} \cdot \frac{1}{2} G^{i}-\eta^{6} \frac{1}{2} H^{i}, \\
= & \frac{1}{2} A^{i}-\eta^{2} \cdot \frac{1}{4}\left(B^{i-1}+B^{i+1}\right)+\eta^{4} \cdot \frac{3}{16}\left(C^{i-2}+4 C^{i}+C^{i+2}\right)-\eta^{6} \cdot \frac{5}{32}\left(D^{i-3}+9 D^{i-1}+9 D^{i+1}+D^{i+3}\right) ; \\
& \eta^{2} \cdot \frac{1}{2} B^{i-1}-\eta^{4} \cdot L^{i}+\eta^{6} S^{i},=\eta^{2} \cdot \frac{1}{2} B^{i+1}-\eta^{4}\left(\frac{3}{4} C^{i-2}+C^{i}\right)+\eta^{6} \cdot \frac{15}{16}\left(D^{i-3}+3 D^{i-1}+D^{i+1}\right) ; \\
& \eta^{4} \cdot \frac{3}{8} C^{i-2}-\eta^{6} T^{i},=\eta^{4} \cdot \frac{3}{8} C^{i-2}-\eta^{6} \cdot \frac{15}{16}\left(D^{i-3}+D^{i-1}\right) ;
\end{aligned}
$$

and

$$
\eta^{6} \cdot \frac{5}{16} D^{i-3} \text {. }
$$

Writing herein $j$ in place of $i$, and for $A^{j}, B^{j-1}$, \&c., the equal values $A^{-j}, B^{-j+1}$, \&c., we have precisely the foregoing coefficients $D(j,-j), \ldots D(j,-j+6)$.

\section{The Development in Powers of $e, e^{\prime}$.}

The complete expression of the reciprocal of the distance is obtained from

$$
\begin{aligned}
& D(j,-j) \quad \cos \left(j U-j U^{\prime}\right) \\
+ & 2 D(j,-j+2) \cos \left(j U+(-j+2) U^{\prime}\right) \\
+ & 2 D(j,-j+4) \cos \left(j U+(-j+4) U^{\prime}\right) \\
+ & 2 D(j,-j+6) \cos \left(j U+(-j+6) U^{\prime}\right),
\end{aligned}
$$

by writing therein for $r, r^{\prime}, U, U^{\prime}$, instead of the circular, the elliptic values, that is the values

$$
\begin{array}{ll}
r=a \operatorname{elqr}(e, L-\Pi) & =a(1+x), \\
r^{\prime}=a^{\prime} \operatorname{elqr}\left(e^{\prime}, L^{\prime}-\Pi^{\prime}\right) \quad, & =a^{\prime}\left(1+x^{\prime}\right), \\
U=\Pi-\Theta+\operatorname{elta}(e, L-\Pi), & =\Pi-\Theta+f, \\
U^{\prime}=\Pi^{\prime}-\Theta^{\prime}+\operatorname{elta}\left(e^{\prime}, L^{\prime}-\Pi^{\prime}\right), & =\Pi^{\prime}-\Theta^{\prime}+f^{\prime}
\end{array}
$$

$L, \Pi, \Theta$ the mean longitude in orbit, longitude of perihelion in orbit, and longitude of node; and the like for $L^{\prime}, \Pi^{\prime}, \Theta^{\prime}$; "elqr" = elliptic quotient radius, "elta"= elliptic true anomaly; or, what is the same thing, if we write elta $(e, L-\Pi)=L-\Pi+\operatorname{eltt}(e, L-\Pi)$, and the like for elta $\left(e^{\prime}, L^{\prime}-\Pi^{\prime}\right)$, then

$$
\begin{aligned}
& U=L-\Theta+\operatorname{eltt}(e, L-\Pi),=L-\Theta+y, \\
& U^{\prime}=L^{\prime}-\Theta^{\prime}+\operatorname{eltt}\left(e^{\prime}, L^{\prime}-\Pi^{\prime}\right),=L^{\prime}-\Theta^{\prime}+y^{\prime} .
\end{aligned}
$$


The process for doing this is explained, First Part, pp. 205-207, [214], viz., writing $r=a(1+x), r^{\prime}=a^{\prime}\left(1+x^{\prime}\right)$, and restoring $j^{\prime}$ (instead of its value $-j, \ldots-j+6$, as the case may be), we have a general term

$$
\frac{1}{\Pi \alpha \Pi \alpha^{\prime}} a^{a} a^{\prime \alpha^{\prime}}\left(\frac{d}{d a}\right)^{a}\left(\frac{d}{d a}\right)^{\alpha^{\prime}} \cdot D\left(j, j^{\prime}\right) \cdot x^{a} x^{\prime a^{\prime}} \cos \left[j(\Pi-\Theta+f)+j^{\prime}\left(\Pi^{\prime}-\Theta^{\prime}+f^{\prime}\right)\right],
$$

where $D\left(j, j^{\prime}\right)$ now denotes the value obtained by writing $a$, $a^{\prime}$ in place of $r, r^{\prime}$ and $f, f^{\prime}$ are the true anomalies elta $(e, L-\Pi)$ and elta $\left(e^{\prime}, L^{\prime}-\Pi^{\prime}\right)$. And the second factor, $x^{a} x^{\prime \alpha^{\prime}}$ into the cosine, is given as a series

$$
\Sigma \Sigma\left([\cos ]^{i}+[\sin ]^{i}\right)\left([\cos ]^{\prime}+[\sin ]^{i^{\prime}}\right) \cos \left[i(L-\Pi)+i^{\prime}\left(L^{\prime}-\Pi^{\prime}\right)+j(\Pi-\Theta)-j^{\prime}\left(\Pi^{\prime}-\Theta^{\prime}\right)\right],
$$

where $[\cos ]^{i},[\sin ]^{i}$ are functions of $e,[\cos ]^{i^{\prime}},[\sin ]^{i^{\prime}}$ functions of $e^{\prime}$. Or, what is better, the term $x^{a} x^{\prime \alpha^{\prime}}$ into the cosine may be written $x^{a} x^{\prime a^{\prime}} \cos \left[j(L-\Theta+y)+j^{\prime}\left(L^{\prime}-\Theta^{\prime}+y^{\prime}\right)\right]$, and the expansion then is

$$
\Sigma \Sigma\left([\cos ]^{i}+[\sin ]^{i}\right)\left([\cos ]^{i^{\prime}}+[\sin ]^{i^{\prime}}\right) \cos \left[i(L-\Pi)+i^{\prime}\left(L^{\prime}-\Pi^{\prime}\right)+j(L-\Theta)+j^{\prime}\left(L^{\prime}-\Theta^{\prime}\right)\right],
$$

where as before $[\cos ]^{i},[\sin ]^{i}$ are functions of $e,[\cos ]^{i^{\prime}},[\sin ]^{i^{\prime}}$ are the same functions of $e^{\prime}$, viz. the $e$-functions are those given in the two "datum-tables" $\left(x^{0} \ldots x^{7}\right) \cos j y$ and $\left(x^{0} \ldots x^{7}\right) \sin j y$, taken from Leverrier, which I have given in my "Tables of the Developments of Functions in the Theory of Elliptic Motion," Memoirs R.A.S. vol. xxix. (1861), pp. 191-306, [216]. In order to better show which are the symbols referred to, we may, instead of $[\cos ]^{i}$, \&c., write $\left[x^{\alpha} \cos j y\right]^{i}$, \&c., the formula will then be

$$
\begin{aligned}
& x^{\alpha} x^{\prime \alpha^{\prime}} \cos \left[j(L-\Theta+y)+j^{\prime}\left(L^{\prime}-\Theta^{\prime}+y^{\prime}\right)\right]= \\
& \sum \Sigma\left(\left[x^{\alpha} \cos j y\right]^{i}+\left[x^{\alpha} \sin j y\right]^{i}\right)\left(\left[x^{\prime \alpha^{\prime}} \cos j^{\prime} y^{\prime}\right]^{i^{\prime}}+\left[x^{\prime \alpha^{\prime}} \sin j^{\prime} y^{\prime}\right]^{i^{\prime}}\right) \\
& \times \cos \left[i(L-\Pi)+i^{\prime}\left(L^{\prime}-\Pi^{\prime}\right)+j(L-\Theta)+j^{\prime}\left(L^{\prime}-\Theta^{\prime}\right)\right] ;
\end{aligned}
$$

and if we attribute to $i, i^{\prime}$ any given values, that is, attend to any particular multiple cosine,

$$
\cos \left[i(L-\Pi)+i^{\prime}\left(L^{\prime}-\Pi^{\prime}\right)+j(L-\Theta)+j^{\prime}\left(L^{\prime}-\Theta^{\prime}\right)\right]
$$

the coefficient hereof will be

$$
\Sigma_{\Pi \alpha \Pi \alpha^{\prime}} a^{a}\left(\frac{d}{d a}\right)^{a} \alpha^{\prime \alpha^{\prime}}\left(\frac{d}{d a}\right)^{\alpha^{\prime}} D\left(j, j^{\prime}\right) \cdot\left(\left[x^{\alpha} \cos j y\right]^{i}+\left[x^{\alpha} \sin j y\right]^{i}\right)\left(\left[x^{\prime} \alpha^{\prime} \cos j^{\prime} y^{\prime}\right]^{i^{\prime}}+\left[x^{\prime \alpha^{\prime}} \sin j^{\prime} y^{\prime}\right]^{i^{\prime}}\right),
$$

where $\alpha, \alpha^{\prime}$ each extend from zero to infinity, but to obtain the expression up to a given order $p$ in $e$, $e^{\prime}$, we take only the values up to $\alpha+\alpha^{\prime}=p$.

Particular, Case.

Thus, for instance, in $\cos \left[j(L-\Theta)-j^{\prime}\left(L^{\prime}-\Theta^{\prime}\right)\right]$ the terms independent of $e^{\prime}$ are

$$
\begin{aligned}
& D(j,-j)\left\{\left[x^{0} \cos j y\right]^{0}+\left[x^{0} \sin j y\right]^{0}\right\} \\
& +\frac{1}{1} a\left(\frac{d}{d a}\right) D\left(j,-j^{\prime}\right)\left\{\left[x^{\prime} \cos j y\right]^{0}+\left[x^{\prime} \sin j y\right]^{0}\right\} \\
& +\frac{1}{1.2} a^{2}\left(\frac{d}{d a}\right)^{2} D(j,-j)\left\{\left[x^{2} \cos j y\right]^{0}+\left[x^{2} \sin j y\right]^{0}\right\} \\
& +\& c .
\end{aligned}
$$


which, observing that in the present case the sine terms vanish, is

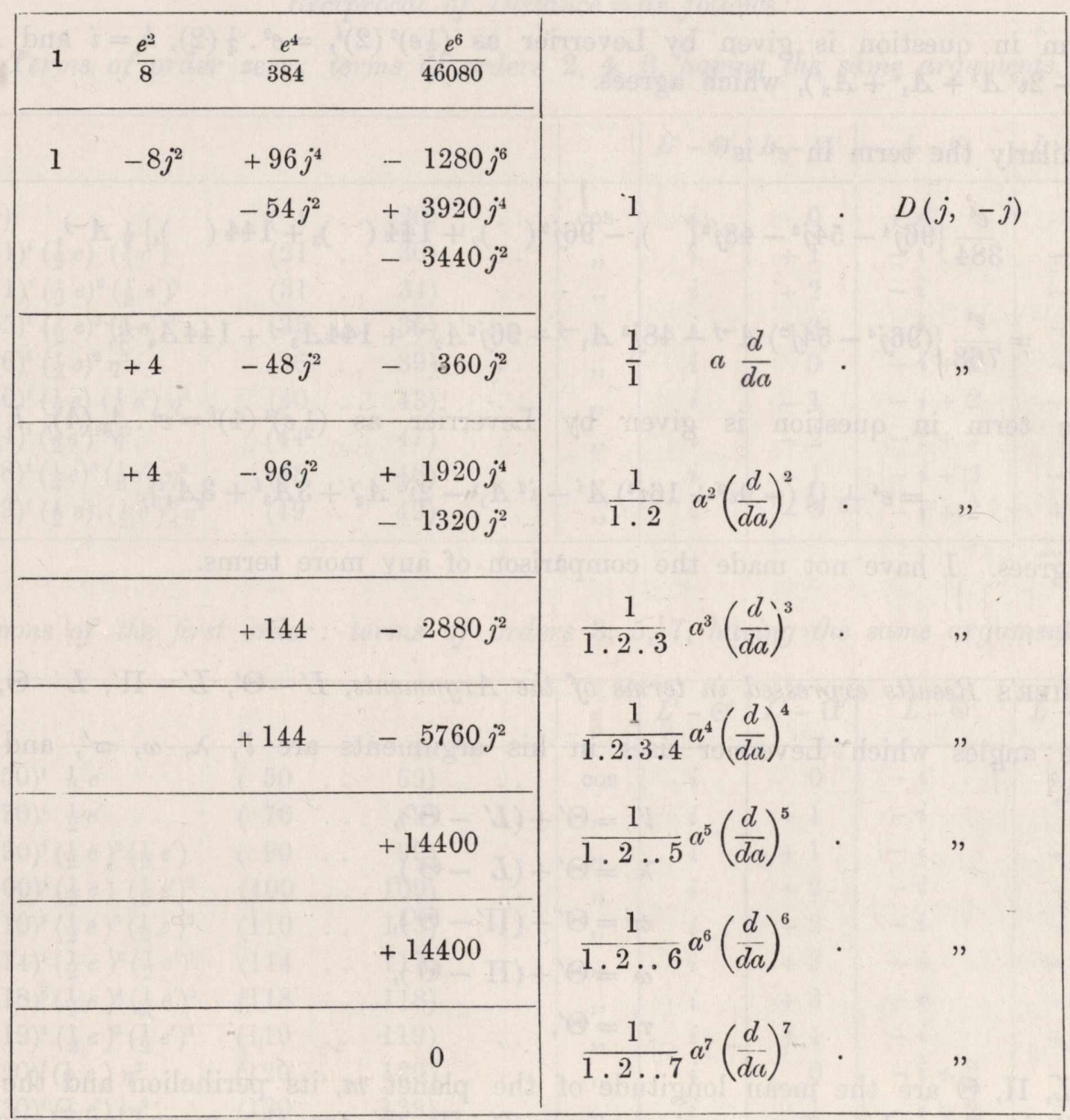

viz. the term in $e^{2}$ is

$$
e^{2}\left\{-j^{2}+\frac{1}{2} a \frac{d}{d a}+\frac{1}{4} a^{2}\left(\frac{d}{d a}\right)^{2}\right\} D(j,-j):
$$

viz. writing $\eta=0$, and therefore $D(j,-j)=\frac{1}{2} A^{-j}$, the term in $e^{2}$ is

$$
e^{2}\left\{-j^{2}+\frac{1}{2} a \frac{d}{d a}+\frac{1}{4} a^{2}\left(\frac{d}{d a}\right)^{2}\right\}^{\frac{1}{2}} A^{-j}
$$

which, conformably with Leverrier's subscript notation

$$
A_{1}^{i}=\frac{1}{1} a \frac{d}{d a} A^{i}, A_{2}^{i}=\frac{1}{1.2} a^{2}\left(\frac{d}{d a}\right)^{2} A^{i}, \text { \&c. }
$$


I write

$$
e^{2}\left\{-j^{2}+\frac{1}{2}()_{1}+\frac{1}{4} 2()_{2}\right\} \frac{1}{2} A^{-j}=e^{2}\left\{-\frac{1}{2} j^{2} A^{-j}+\frac{1}{4} A_{1}^{-j}+\frac{1}{4} A_{2}^{-j}\right\} .
$$

The term in question is given by Leverrier as $\left(\frac{1}{2} e\right)^{2}(2)^{i},=e^{2} \cdot \frac{1}{4}(2), h=i$ and $K^{i}=A^{i}$, $=e^{2} \cdot \frac{1}{4}\left(-2 i^{2} A^{i}+A_{1}^{i}+A_{2}^{i}\right)$, which agrees.

Similarly the term in $e^{4}$ is

$$
\begin{aligned}
& \frac{e^{4}}{384}\left\{96 j^{4}-54 j^{2}-48 j^{2}()_{1}-96 j^{2}()_{2}+144()_{3}+144()_{4}\right\} \frac{1}{2} A^{-j}, \\
= & \frac{e^{4}}{768}\left\{\left(96 j^{4}-54 j^{2}\right) A^{-j}-48 j^{2} A_{1}^{-j}-96 j^{2} A_{2}^{-j}+144 A_{3}^{-j}+144 A_{4}^{-j}\right\},
\end{aligned}
$$

and the term in question is given by Leverrier as $\left(\frac{1}{2} e\right)^{4}(4)^{i}=e^{4} \cdot \frac{1}{16}(4), h=i$ and $K^{i}=A^{i}$,

$$
=e^{4} \frac{1}{16}\left\{\frac{1}{8}\left(-9 i^{2}+16 i^{4}\right) A^{i}-i^{2} A_{1}^{i}-2 i^{2} A_{2}^{i}+3 A_{3}^{i}+3 A_{4}^{i}\right\},
$$

which agrees. I have not made the comparison of any more terms.

LEVERRIER's Results expressed in terms of the Arguments, $L^{\prime}-\Theta^{\prime}, L^{\prime}-\Pi^{\prime}, L-\Theta, L-\Pi$.

The angles which Leverrier uses in his arguments are $l^{\prime}, \lambda, \omega, \varpi^{\prime}$, and $\tau^{\prime}$, viz. we have,

$$
\begin{aligned}
& l^{\prime}=\Theta^{\prime}+\left(L^{\prime}-\Theta^{\prime}\right), \\
& \lambda=\Theta^{\prime}+(L-\Theta), \\
& \varpi^{\prime}=\Theta^{\prime}+\left(\Pi^{\prime}-\Theta^{\prime}\right), \\
& \omega=\Theta^{\prime}+(\Pi-\Theta), \\
& \tau^{\prime}=\Theta^{\prime},
\end{aligned}
$$

where $L, \Pi, \Theta$ are the mean longitude of the planet $m$, its perihelion and the mutual node, all in the orbit of $m$; and similarly $L^{\prime}, \Pi^{\prime}, \Theta^{\prime}$ are the mean longitude of the planet $m^{\prime}$, of its perihelion and of the mutual node, all in the orbit of $m^{\prime}$. On substituting the foregoing values of $l^{\prime}, \lambda$, \&c., $\Theta^{\prime}$, as it should do, disappears, and the arguments are all of them linear functions of $L^{\prime}-\Theta^{\prime}, \Pi^{\prime}-\Theta^{\prime}, L-\Theta, \Pi-\Theta$; or, if we please, of $L^{\prime}-\Theta^{\prime}, L^{\prime}-\Pi^{\prime}, L-\Theta, L-\Pi$, that is of the distances of each planet from its own perihelion and from the mutual node. It is, I think, convenient to use these last angular distances, and accordingly in Leverrier's arguments, I write,

$$
\begin{aligned}
& l^{\prime}=\Theta^{\prime}+\left(L^{\prime}-\Theta^{\prime}\right), \\
& \lambda=\Theta^{\prime} \cdot \quad \cdot \quad \cdot(L-\Theta), \\
& \varpi^{\prime}=\Theta^{\prime}+\left(L^{\prime}-\Theta^{\prime}\right)-\left(L^{\prime}-\Pi^{\prime}\right), \\
& \omega=\Theta^{\prime} . \quad \cdot \quad+(L-\Theta)-(L-\Pi), \\
& \tau^{\prime}=\Theta^{\prime}
\end{aligned}
$$


and for the purpose of reference form as it were an Index to his result as follows:

\section{Reciprocal of Distance $=$ as follows :}

Terms of order zero: terms of orders 2, 4, 6, having the same arguments.

\begin{tabular}{|c|c|c|c|c|c|c|c|c|}
\hline & & & & & $L^{\prime}-\Theta^{\prime}$ & $L^{\prime}-\Pi^{\prime}$ & $L-\Theta$ & $L-\Pi$ \\
\hline$(1)^{i}$ & $(1$ & . $\quad 20$ ) & . & $\cos$ & $i$ & 0 & $-i$ & 0 \\
\hline$(21)^{i}\left(\frac{1}{2} e\right)\left(\frac{1}{2} e^{\prime}\right)$ & $(21$ & . 30$)$ & . & " & $i$ & +1 & $-i$ & -1 \\
\hline$(31)^{i}\left(\frac{1}{2} e\right)^{2}\left(\frac{1}{2} e^{\prime}\right)^{2}$ & (31 & . 34$)$ & . & " & $i$ & +2 & $-i$ & -2 \\
\hline$(35)^{i}\left(\frac{1}{2} e\right)^{3}\left(\frac{1}{2} e^{\prime}\right)^{3}$ & $(35$ & . 35$)$ & . & $"$ & $i$ & +3 & $-i$ & -3 \\
\hline$(36)^{i}\left(\frac{1}{2} e\right)^{2} \eta^{2}$ & (36 & . . 39) & & , & $i$ & 0 & $-i+2$ & -2 \\
\hline$(40)^{i}\left(\frac{1}{2} e\right)\left(\frac{1}{2} e^{\prime}\right) \eta^{2-}$ & $(40$ & . 43$)$ & . & , & $i$ & -1 & $-i+2$ & -1 \\
\hline$(44)^{i}\left(\frac{1}{2} e^{\prime}\right)^{2} \eta^{2}$ & $(44$ & $\ldots \quad 47)$ & . & , & $i$ & -2 & $-i+2$ & 0 \\
\hline$(48)^{i}\left(\frac{1}{2} e\right)^{3}\left(\frac{1}{2} e^{\prime}\right) \eta^{2}$ & $(48$ & . 48$)$ & . & ", & $i$ & +1 & $-i+2$ & -3 \\
\hline$(49)^{i}\left(\frac{1}{2} e\right)\left(\frac{1}{2} e^{\prime}\right)^{3} \eta^{2}$ & $(49$ & . . 49) & & ", & $i$ & -3 & $-i+2$ & +1 \\
\hline
\end{tabular}

Terms of the first order: terms of orders 3, 5, 7, having the same arguments.

\begin{tabular}{|c|c|c|c|c|c|c|c|c|}
\hline & & & & & $L^{\prime}-\Theta^{\prime}$ & $L^{\prime}-\Pi^{\prime}$ & $L-\Theta$ & $L-\Pi$ \\
\hline$(50)^{i} \frac{1}{2} e$ & ( 50 & 69) & . & $\cos$ & $i$ & 0 & $-i$ & +1 \\
\hline$(70)^{i} \frac{1}{2} e^{\prime}$ & $(70$ & . $\quad 89)$ & . & , & $i$ & +1 & $-i$ & 0 \\
\hline$(90)^{i}\left(\frac{1}{2} e\right)^{2}\left(\frac{1}{2} e^{\prime}\right)$ & ( 90 & . $\quad 99)$ & . & $"$ & $i$ & +1 & $-i$ & -2 \\
\hline$(100)^{i}\left(\frac{1}{2} e\right)\left(\frac{1}{2} e^{\prime}\right)^{2}$ & $(100$ & . 109) & . & $"$ & $i$ & +2 & $-i$ & -1 \\
\hline$(110)^{i}\left(\frac{1}{2} e\right)^{3}\left(\frac{1}{2} e^{\prime}\right)^{2}$ & (110 & . 113$)$ & .. & , & $i$ & +2 & $-i$ & -3 \\
\hline$(114)^{i}\left(\frac{1}{2} e\right)^{2}\left(\frac{1}{2} e^{\prime}\right)^{3}$ & (114 & . . 117) & . & $"$ & $i$ & +3 & $-i$ & -2 \\
\hline$(118)^{i}\left(\frac{1}{2} e\right)^{4}\left(\frac{1}{2} e^{\prime}\right)^{3}$ & (118 & . . 118) & & , & $i$ & +3 & $-i$ & -4 \\
\hline$(119)^{i}\left(\frac{1}{2} e\right)^{3}\left(\frac{1}{2} e^{\prime}\right)^{4}$ & (119 & . 119) & . & " & $i$ & +4 & $-i$ & -3 \\
\hline$(120)^{i}\left(\frac{1}{2} e\right) \eta^{2}$ & (120 & . . 129) & . & $"$ & $i$ & 0 & $-i+2$ & -1 \\
\hline$(130)^{i}\left(\frac{1}{2} e^{\prime}\right) \eta^{2}$ & (130 & .. 139) & & $"$ & $i$ & -1 & $-i+2$ & 0 \\
\hline$(140)^{i}\left(\frac{1}{2} e\right)^{3} \eta^{2}$ & $(140$ & . 143 ) & . & $"$ & $i$ & 0 & $-i+2$ & -3 \\
\hline$(144)^{i}\left(\frac{1}{2} e\right)^{2}\left(\frac{1}{2} e^{\prime}\right) \eta^{2}$ & (144 & .. 147) & . & " & $i$ & +1 & $-i+2$ & -2 \\
\hline$(148)^{i}\left(\frac{1}{2} e\right)^{2}\left(\frac{1}{2} e^{\prime}\right) \eta^{2}$ & $(148$ & . . 151) & . & , & $i$ & -1 & $-i+2$ & -2 \\
\hline$(152)^{i}\left(\frac{1}{2} e\right)\left(\frac{1}{2} e^{\prime}\right)^{2} \eta^{2}$ & $(152$ & . . 155) & . & " & $i$ & -2 & $-i+2$ & -1 \\
\hline$(156)^{i} \frac{1}{2} e \quad\left(\frac{1}{2} e^{\prime}\right)^{2} \eta^{2}$ & $(156$ & .. 159) & & " & $i$ & -2 & $-i+2$ & +1 \\
\hline$(160)^{i}\left(\frac{1}{2} e^{\prime}\right)^{3} \eta^{2}$ & $(160$ & . . 163) & . & $"$ & $i$ & -3 & $-i+2$ & 0 \\
\hline$(164)^{i}\left(\frac{1}{2} e\right)^{4}\left(\frac{1}{2} e^{\prime}\right) \eta^{2}$ & (164 & .. 164) & . & $"$ & $i$ & +1 & $-i+2$ & -4 \\
\hline$(165)^{i}\left(\frac{1}{2} e\right)^{3}\left(\frac{1}{2} e^{\prime}\right)^{2} \eta^{2}$ & $(165$ & .. 165$)$ & & , & $i$ & +2 & $-i+2$ & -3 \\
\hline$(166)^{i}\left(\frac{1}{2} e\right)^{2}\left(\frac{1}{2} e^{\prime}\right)^{3} \eta^{2}$ & $(166$ & . . 166) & . & $"$ & $i$ & -3 & $-i+2$ & +2 \\
\hline$(167)^{i}\left(\frac{1}{2} e\right)\left(\frac{1}{2} e^{\prime}\right)^{4} \eta^{2}$ & (167 & .. 167) & . & $"$ & $i$ & -4 & $-i+2$ & +1 \\
\hline$(168)^{i}\left(\frac{1}{2} e\right)^{3} \eta^{4}$ & $(168$ & .. 168) & . & " & $i$ & 0 & $-i+2$ & -3 \\
\hline$(169)^{i}\left(\frac{1}{2} e\right)^{2}\left(\frac{1}{2} e^{\prime}\right) \eta^{4}$ & $(169$ & . . 169) & & " & $i$ & -1 & $-i+4$ & -2 \\
\hline$(170)^{i}\left(\frac{1}{2} e\right)\left(\frac{1}{2} e^{\prime}\right)^{2} \eta^{4}$ & $(170$ & .. 170) & . & $"$ & $i$ & -2 & $-i+4$ & -1 \\
\hline$(171)^{i}\left(\frac{1}{2} e^{\prime}\right)^{3} \eta^{4}$ & (171 & . 171) & . & $"$ & $i$ & -3 & $-i+4$ & 0 \\
\hline
\end{tabular}


Terms of second order: terms of orders 4, 6, having the same arguments.

\begin{tabular}{|c|c|c|c|c|c|c|c|c|}
\hline & & & & & $L^{\prime}-\Theta^{\prime}$ & $L^{\prime}-\Pi^{\prime}$ & $L-\Theta$ & $L-\Pi$ \\
\hline$(172)^{i}\left(\frac{1}{2} e\right)^{2}$ & $(172$ & .. 181) & . & $\cos$ & $i$ & 0 & $-i$ & +2 \\
\hline$(182)^{i}\left(\frac{1}{2} e\right)\left(\frac{1}{2} e^{\prime}\right)$ & $(182$ & .. 191) & . & $"$ & $i$ & +1 & $-i$ & +1 \\
\hline$(192)^{i}\left(\frac{1}{2} e^{\prime}\right)^{2}$ & $(192$ & . 201) & . & $"$ & $i$ & +2 & $-i$ & 0 \\
\hline$(202)^{i}\left(\frac{1}{2} e\right)^{3}\left(\frac{1}{2} e^{\prime}\right)$ & $(202$ & . . 205) & . & " & $i$ & +1 & $-i$ & -3 \\
\hline$(206)^{i}\left(\frac{1}{2} e\right)\left(\frac{1}{2} e^{\prime}\right)^{3}$ & $(206$ & . 209) & . & $"$ & $i$ & +3 & $-i$ & -1 \\
\hline$(210)^{i}\left(\frac{1}{2} e\right)^{4}\left(\frac{1}{2} e^{\prime}\right)^{2}$ & $(210$ & . 210) & . & " & $i$ & +2 & $-i$ & -4 \\
\hline$(211)^{i}\left(\frac{1}{2} e\right)^{2}\left(\frac{1}{2} e^{\prime}\right)^{4}$ & $(211$ & .. 211) & $\cdots$ & $”$ & $i$ & +4 & $-i$ & -2 \\
\hline$(212)^{i} \eta^{2}$ & $(212$ & . 221) & $\cdots$ & " & $i$ & 0 & $-i+2$ & 0 \\
\hline$(222)^{i}\left(\frac{1}{2} e\right)\left(\frac{1}{2} e^{\prime}\right) \eta^{2}$ & $(222$ & . $\quad 225)$ & $\cdots$ & $"$ & $i$ & +1 & $-i+2$ & -1 \\
\hline$(226)^{i}\left(\frac{1}{2} e\right)\left(\frac{1}{2} e^{\prime}\right) \eta^{2}$ & $(226$ & . . 229) & . & $"$ & $i$ & -1 & $-i+2$ & +1 \\
\hline$(230)^{i}\left(\frac{1}{2} e\right)^{4} \eta^{2}$ & $(230$ & . 230 ) & . & $"$ & $i$ & 0 & $-i+2$ & -4 \\
\hline$(231)^{i}\left(\frac{1}{2} e\right)^{3}\left(\frac{1}{2} e^{\prime}\right) \eta^{2}$ & $(231$ & . 2 231) & $\cdots$ & $"$ & $i$ & -1 & $-i+2$ & -3 \\
\hline$(232)^{i}\left(\frac{1}{2} e\right)^{2}\left(\frac{1}{2} e^{\prime}\right)^{2} \eta^{2}$ & $(232$ & . 232). & $\cdots$ & ” & $i$ & -2 & $-i+2$ & -2 \\
\hline$(233)^{i}\left(\frac{1}{2} e\right)\left(\frac{1}{2} e^{\prime}\right)^{3} \eta^{2}$ & $(233$ & . . 233) & . & ” & $i$ & -3 & $-i+2$ & -1 \\
\hline$(234)^{i}\left(\frac{1}{2} e^{\prime}\right)^{4} \eta^{2}$ & $(234$ & . 234) & . & " & $i$ & -4 & $-i+2$ & 0 \\
\hline$(235)^{i}\left(\frac{1}{2} e^{\prime}\right)^{2}\left(\frac{1}{2} e^{\prime}\right)^{2} \eta^{2}$ & $(235$ & . 235) & $\cdots$ & $"$ & $i$ & +2 & $-i+2$ & -2 \\
\hline$(236)^{i}\left(\frac{1}{2} e\right)^{2}\left(\frac{1}{2} e^{\prime}\right)^{2} \eta^{2}$ & $(236$ & . 236 ) & $\cdots$ & ", & $i$ & -2 & $-i+2$ & +2 \\
\hline$(237)^{i}\left(\frac{1}{2} e\right)^{2} \eta^{4}$ & $(237$ & . 237) & $\cdots$ & ” & $i$ & 0 & $-i+4$ & -2 \\
\hline$(238)^{i}\left(\frac{1}{2} e\right)\left(\frac{1}{2} e^{\prime}\right) \eta^{4}$ & $(238$ & . . 238) & & $"$ & $i$ & -1 & $-i+4$ & -1 \\
\hline$(239)^{i}\left(\frac{1}{2} e^{\prime}\right)^{2} \eta^{4}$ & $(239$ & . 239) & . & ", & $i$ & -2 & $-i+4$ & 0 \\
\hline
\end{tabular}

Terms of third order: terms of orders 5, 7, having the same arguments.

\begin{tabular}{|c|c|c|c|c|c|c|c|c|}
\hline & & & & & $L^{\prime}-\Theta^{\prime}$ & $L^{\prime}-\Pi^{\prime}$ & $L-\Theta$ & $L-\Pi$ \\
\hline$(240)^{i}\left(\frac{1}{2} e\right)^{3}$ & $(240$ & . . 249) & . & $\cos$ & $i$ & 0 & $-i$ & +3 \\
\hline$(250)^{i}\left(\frac{1}{2} e\right)^{2}\left(\frac{1}{2} e^{\prime}\right)$ & $(250$ & 259) & $\cdots$ & $"$ & $i$ & +1 & $-i$ & +2 \\
\hline$(260)^{i}\left(\frac{1}{2} e\right)\left(\frac{1}{2} e^{\prime}\right)^{2}$ & $(260$ & . 269) & . & $"$ & $i$ & +2 & $-i$ & +1 \\
\hline$(270)^{i}\left(\frac{1}{2} e^{\prime}\right)^{3}$ & $(270$ & 279) & . & " & $i$ & +3 & $-i$ & 0 \\
\hline
\end{tabular}


Terms of third order (concluded):

\begin{tabular}{|c|c|c|c|c|c|c|c|c|}
\hline & & & & & & & & \\
\hline & & & & & $L^{\prime}-\Theta^{\prime}$ & $L^{\prime}-\Pi^{\prime}$ & $L-\Theta$ & $L-\Pi$ \\
\hline$(280)^{i}\left(\frac{1}{2} e\right)^{4}\left(\frac{1}{2} e^{\prime}\right)$ & $(280$ & . $\quad 283)$ & . & $\cos$ & $i$ & +1 & $-i$ & -4 \\
\hline$(284)^{i}\left(\frac{1}{2} e\right)\left(\frac{1}{2} e^{\prime}\right)^{4}$ & $(284$ & . 287) & . & $"$ & $i$ & +4 & $-i$ & -1 \\
\hline$(288)^{i}\left(\frac{1}{2} e\right)^{3}\left(\frac{1}{2} e^{\prime}\right)^{2}$ & $(288$ & . . 289) & . & " & $i$ & +2 & $-i$ & -5 \\
\hline$(290)^{i}\left(\frac{1}{2} e\right)^{2}\left(\frac{1}{2} e^{\prime}\right)^{3}$ & $(290$ & . . 299) & . & , & $i$ & 0 & $-i+2$ & +1 \\
\hline$(300)^{i}\left(\frac{1}{2} e^{\prime}\right) \eta^{2}$ & $(300$ & . 309$)$ & . & , & $i$ & +1 & $-i+2$ & 0 \\
\hline$(310)^{i}\left(\frac{1}{2} e\right)^{2}\left(\frac{1}{2} e^{\prime}\right) \eta^{2}$ & $(310$ & . 313$)$ & . & , & $i$ & -1 & $-i+2$ & +2 \\
\hline$(314)^{i}\left(\frac{1}{2} e\right)\left(\frac{1}{2} e^{\prime}\right)^{2} \eta^{2}$ & $(314$ & . 317$)$ & . & ", & $i$ & +2 & $-i+2$ & -1 \\
\hline$(318)^{i}\left(\frac{1}{2} e\right)^{3} \eta^{2}$ & $(318$ & . 318$)$ & . & ", & $i$ & 0 & $-i+2$ & -5 \\
\hline$(319)^{i}\left(\frac{1}{2} e\right)^{4}\left(\frac{1}{2} e^{\prime}\right) \eta^{2}$ & $(319$ & . 319$)$ & $\cdots$ & ", & $i$ & -1 & $-i+2$ & -4 \\
\hline$(320)^{i}\left(\frac{1}{2} e\right)^{3}\left(\frac{1}{2} e^{\prime}\right)^{2} \eta^{2}$ & $(320$ & . . 320$)$ & . . & , & $i$ & -2 & $-i+2$ & -3 \\
\hline$(321)^{i}\left(\frac{1}{2} e\right)^{2}\left(\frac{1}{2} e^{\prime}\right)^{3} \eta^{2}$ & $(321$ & .. 321) & . & $"$ & $i$ & -3 & $-i+2$ & -2 \\
\hline$(322)^{i}\left(\frac{1}{2} e\right)\left(\frac{1}{2} e^{\prime}\right)^{4} \eta^{2}$ & $(322$ & . 322$)$ & 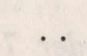 & , & $i$ & -4 & $-i+2$ & -1 \\
\hline$(323)^{i}\left(\frac{1}{2} e^{\prime}\right)^{5} \eta^{2}$ & $(323$ & .. 323) & . & ", & $i$ & -5 & $-i+2$ & 0 \\
\hline$(324)^{i}\left(\frac{1}{2} e\right)^{3}\left(\frac{1}{2} e^{\prime}\right)^{2} \eta^{2}$ & $(324$ & . . 324) & . & ", & $i$ & -2 & $-i+2$ & +3 \\
\hline$(325)^{i}\left(\frac{1}{2} e\right)^{2}\left(\frac{1}{2} e^{\prime}\right)^{3} \eta^{2}$ & $(325$ & . . 325) & . & ” & $i$ & +3 & $-i+2$ & -2 \\
\hline$(326)^{i}\left(\frac{1}{2} e\right) \eta^{4}$ & $(326$ & . 329$)$ & 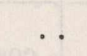 & ", & $i$ & 0 & $-i+4$ & -1 \\
\hline$(330)^{i}\left(\frac{1}{2} e^{\prime}\right) \eta^{4}$ & $(330$ & . $\quad 333)$ & $\cdots$ & " & $i$ & -1 & $-i+4$ & 0 \\
\hline$(334)^{i}\left(\frac{1}{2} e\right)^{2}\left(\frac{1}{2} e^{\prime}\right) \eta^{4}$ & $(334$ & .. 334) & . & , & $i$ & +1 & $-i+4$ & -2 \\
\hline$(335)^{i}\left(\frac{1}{2} e\right)\left(\frac{1}{2} e^{\prime}\right)^{2} \eta^{4}$ & $(335$ & . 335$)$ & & , & $i$ & -2 & $-i+4$ & +1 \\
\hline
\end{tabular}

Terms of fourth order: terms of order 6, and of same argument.

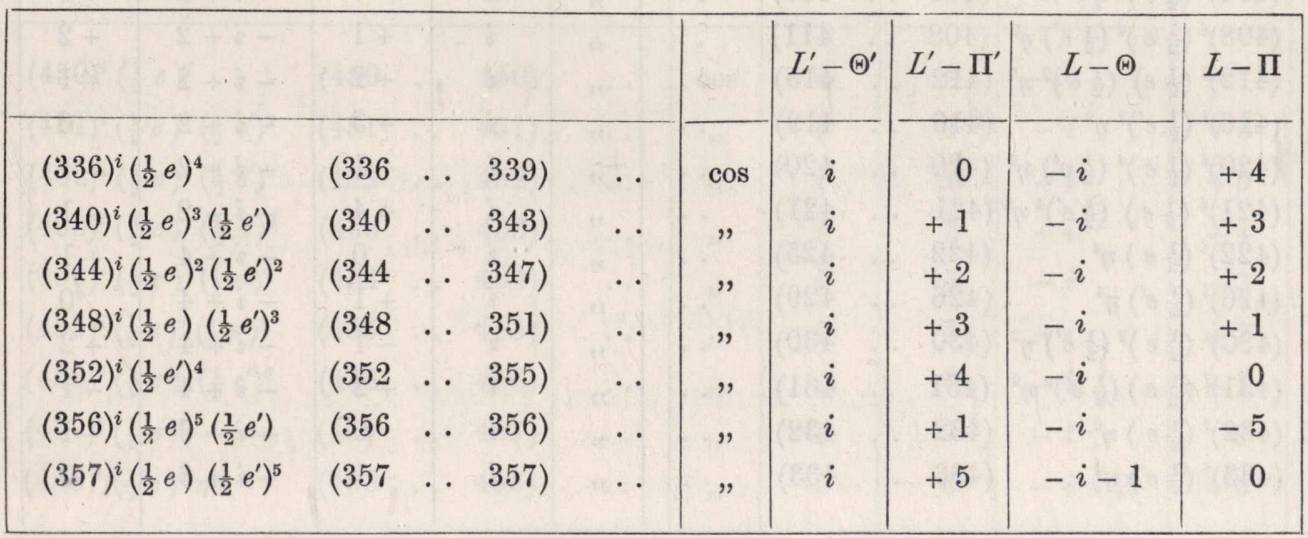

C. VII. 
Terms of fourth order (concluded):

\begin{tabular}{|lllll|l|l|l|l|r|}
\hline & & & & & & $L^{\prime}-\Theta^{\prime}$ & $L^{\prime}-\Pi^{\prime}$ & $L-\Theta$ & $L-\Pi$ \\
\hline$(358)^{i}\left(\frac{1}{2} e\right)^{2} \eta^{2}$ & $(358$ & $\ldots$ & $358)$ & $\ldots$ & cos & $i$ & 0 & $-i+2$ & +2 \\
$(362)^{i}\left(\frac{1}{2} e\right)\left(\frac{1}{2} e^{\prime}\right) \eta^{2}$ & $(362$ & $\ldots$ & $364)$ & $\ldots$ & $\#$ & $i$ & +1 & $-i+2$ & +1 \\
$(366)^{i}\left(\frac{1}{2} e^{\prime}\right)^{2} \eta^{2}$ & $(366$ & $\ldots$ & $369)$ & $\ldots$ & $\#$ & $i$ & +2 & $-i+2$ & 0 \\
$(370)^{i}\left(\frac{1}{2} e\right)^{3}\left(\frac{1}{2} e^{\prime}\right) \eta^{2}$ & $(370$ & $\ldots$ & $370)$ & $\ldots$ & $\#$ & $i$ & -1 & $-i+2$ & +3 \\
$(371)^{i}\left(\frac{1}{2} e\right)\left(\frac{1}{2} e^{\prime}\right)^{3} \eta^{2}$ & $(371$ & $\ldots$ & $371)$ & $\ldots$ & $\#$ & $i$ & +3 & $-i+2$ & -1 \\
$(372)^{i} \eta^{4}$ & $(372$ & $\ldots$ & $375)$ & $\ldots$ & $\#$ & $i$ & 0 & $-i+4$ & 0 \\
$(376)^{i}\left(\frac{1}{2} e\right)\left(\frac{1}{2} e^{\prime}\right) \eta^{4}$ & $(376$ & $\ldots$ & $376)$ & $\ldots$ & $\ldots$ & $i$ & +1 & $-i+4$ & -1 \\
$(377)^{i}\left(\frac{1}{2} e\right)\left(\frac{1}{2} e^{\prime}\right) \eta^{4}$ & $(377$ & $\ldots$ & $377)$ & $\ldots$ & $\ldots$ & $i$ & -1 & $-i+4$ & +1 \\
\hline
\end{tabular}

Terms of fifth order: terms of order 7 having the same arguments.

\begin{tabular}{|c|c|c|c|c|c|c|c|c|}
\hline & & (2) & If & & $L^{\prime}-\Theta^{\prime}$ & $L^{\prime}-\Pi^{\prime}$ & $L-\Theta$ & $L-\Pi$ \\
\hline$(378)^{i}\left(\frac{1}{2} e\right)^{5}$ & $(378$ & .. 381) & . & $\cos$ & $i$ & 0 & $-i$ & +5 \\
\hline$(382)^{i}\left(\frac{1}{2} e\right)^{2}\left(\frac{1}{2} e^{\prime}\right)$ & $(382$ & .. 385$)$ & . & $"$ & $i$ & +1 & $-i$ & +4 \\
\hline$(386)^{i}\left(\frac{1}{2} e\right)^{3}\left(\frac{1}{2} e^{\prime}\right)^{2}$ & $(386$ & . $\quad 389)$ & . & , & $i$ & +2 & $-i$ & +3 \\
\hline$(390)^{i}\left(\frac{1}{2} e\right)^{2}\left(\frac{1}{2} e^{\prime}\right)^{3}$ & $(390$ & . . 393) & . & " & $i$ & +3 & $-i$ & +2 \\
\hline$(394)^{i}\left(\frac{1}{2} e\right)\left(\frac{1}{2} e^{\prime}\right)^{4}$ & $(394$ & .. 397) & . & ", & $i$ & +4 & $-i$ & +1 \\
\hline$(398)^{i}\left(\frac{1}{2} e^{\prime}\right)^{5}$ & (398 & .. 401) & . & $"$ & $i$ & +5 & $-i$ & 0 \\
\hline$(402)^{i}\left(\frac{1}{2} e\right)^{6}\left(\frac{1}{2} e^{\prime}\right)$ & $(402$ & . 402$)$ & . & " & $i$ & +1 & $-i$ & -6 \\
\hline$(403)^{i}\left(\frac{1}{2} e\right)\left(\frac{1}{2} e^{\prime}\right)^{6}$ & $(403$ & .. 403) & . & " & $i$ & +6 & $-i$ & -1 \\
\hline$(404)^{i}\left(\frac{1}{2} e\right)^{3} \eta^{2}$ & $(404$ & . 407$)$ & $\ldots$ & $"$ & $i$ & 0 & $-i+2$ & +3 \\
\hline$(408)^{i}\left(\frac{1}{2} e\right)^{2}\left(\frac{1}{2} e^{\prime}\right) \eta^{2}$ & $(408$ & .. 411) & . & " & $i$ & +1 & $-i+2$ & +2 \\
\hline$(412)^{i}\left(\frac{1}{2} e\right)\left(\frac{1}{2} e^{\prime}\right)^{2} \eta^{2}$ & $(412$ & .. 415) & . & $"$ & $i$ & +2 & $-i+2$ & +1 \\
\hline$(416)^{i}\left(\frac{1}{2} e^{\prime}\right)^{3} \eta^{2}$ & $(416$ & .. 419) & $\cdots$ & $"$ & $i$ & +3 & $-i+2$ & 0 \\
\hline$(420)^{i}\left(\frac{1}{2} e\right)^{4}\left(\frac{1}{2} e^{\prime}\right) \eta^{2}$ & $(420$ & .. 420) & . & $"$ & $i$ & -1 & $-i+2$ & +4 \\
\hline$(421)^{i}\left(\frac{1}{2} e\right)\left(\frac{1}{2} e^{\prime}\right)^{4} \eta^{2}$ & $(421$ & .. 421) & $\cdots$ & $"$ & $i$ & +4 & $-i+2$ & -1 \\
\hline$(422)^{i}\left(\frac{1}{2} e\right) \eta^{4}$ & $(422$ & .. 425) & . & $"$ & $i$ & 0 & $-i+4$ & +1 \\
\hline$(426)^{i}\left(\frac{1}{2} e^{\prime}\right) \eta^{4}$ & $(426$ & 429) & . & $"$ & $i$ & +1 & $-i+4$ & 0 \\
\hline$(430)^{i}\left(\frac{1}{2} e\right)^{2}\left(\frac{1}{2} e^{\prime}\right) \eta^{2}$ & $(430$ & .. 430) & $\ldots$ & " & $i$ & -1 & $-i+4$ & +2 \\
\hline$(431)^{i}\left(\frac{1}{2} e\right)\left(\frac{1}{2} e^{\prime}\right)^{2} \eta^{2}$ & $(431$ & .. 431) & . & $"$ & $i$ & +2 & $-i+4$ & -1 \\
\hline$(432)^{i}\left(\frac{1}{2} e\right) \eta^{6}$ & $(432$ & .. 432 ) & $\cdots$ & $"$ & $i$ & 0 & $-i+6$ & -1 \\
\hline$(433)^{i}\left(\frac{1}{2} e^{\prime}\right) \eta^{4}$ & $(433$ & . 433$)$ & & $"$ & $i$ & -1 & $-i+6$ & 0 \\
\hline
\end{tabular}


Terms of sixth order.

\begin{tabular}{|c|c|c|c|c|c|c|c|c|}
\hline t & & & & & $L^{\prime}-\Theta^{\prime}$ & $L^{\prime}-\Pi^{\prime}$ & $L-\Theta$ & $L-\Pi$ \\
\hline$(434)^{i}\left(\frac{1}{2} e\right)^{6}$ & $(434$ & .. 434) & . & $\cos$ & $i$ & 0 & $-i$ & +6 \\
\hline$(435)^{i}\left(\frac{1}{2} e\right)^{5}\left(\frac{1}{2} e^{\prime}\right)$ & $(43 \check{5}$ & . 435$)$ & . & $"$ & $i$ & +1 & $-i$ & +5 \\
\hline$(436)^{i}\left(\frac{1}{2} e\right)^{4}\left(\frac{1}{2} e^{\prime}\right)^{2}$ & $(436$ & . 4 436) & . & $"$ & $i$ & +2 & $-i$ & +4 \\
\hline$(437)^{i}\left(\frac{1}{2} e\right)^{3}\left(\frac{1}{2} e^{\prime}\right)^{3}$ & $(437$ & .. 437) & . & , & $i$ & +3 & $-i$ & +3 \\
\hline$(438)^{i}\left(\frac{1}{2} e\right)^{2}\left(\frac{1}{2} e^{\prime}\right)^{4}$ & $(438$ & . 438$)$ & . & ” & $i$ & +4 & $-i$ & +2 \\
\hline$(439)^{i}\left(\frac{1}{2} e\right)\left(\frac{1}{2} e^{\prime}\right)^{5}$ & $(439$ & . . 439) & & , & $i$ & +5 & $-i$ & +1 \\
\hline$(440)^{i}\left(\frac{1}{2} e^{\prime}\right)^{6}$ & $(440$ & . 440$)$ & . & , & $i$ & +6 & $-i$ & 0 \\
\hline$(441)^{i}\left(\frac{1}{2} e\right)^{4} \eta^{2}$ & $(441$ & . 441$)$ & & $"$ & $i$ & 0 & $-i+2$ & +4 \\
\hline$(442)^{i}\left(\frac{1}{2} e\right)^{3}\left(\frac{1}{2} e^{\prime}\right) \eta^{2}$ & $(442$ & .. 442) & & ” & $i$ & +1 & $-i+2$ & +3 \\
\hline$(443)^{i}\left(\frac{1}{2} e\right)^{2}\left(\frac{1}{2} e^{\prime}\right)^{2} \eta^{2}$ & ${ }^{2}(443$ & . 443$)$ & & 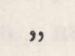 & $i$ & +2 & $-i+2$ & +2 \\
\hline$(444)^{i}\left(\frac{1}{2} e\right)\left(\frac{1}{2} e^{\prime}\right)^{3} \eta^{2}$ & ${ }^{2}(444$ & . . 444) & & $"$ & $i$ & +3 & $-i+2$ & +1 \\
\hline$(445)^{i}\left(\frac{1}{2} e^{\prime}\right)^{4} \eta^{2}$ & $(445$ & .. 445) & . & $"$ & $i$ & +4 & $-i+2$ & 0 \\
\hline$(446)^{i}\left(\frac{1}{2} e\right)^{2} \eta^{4}$ & $(446$ & . 446$)$ & & " & $i$ & 0 & $-i+4$ & +2 \\
\hline$(447)^{i}\left(\frac{1}{2} e\right)\left(\frac{1}{2} e^{\prime}\right) \eta^{4}$ & $(447$ & . 447 ) & 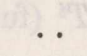 & $"$ & $i$ & +1 & $-i+4$ & +1 \\
\hline$(448)^{i}\left(\frac{1}{2} e^{\prime}\right)^{2} \eta^{4}$ & $(448$ & . 448$)$ & $\cdots$ & " & $i$ & +2 & $-i+4$ & 0 \\
\hline$(449)^{i} \eta^{6}$ & $(449$ & . 449$)$ & $\ldots$ & ", & $i$ & 0 & $-i+6$ & 0 \\
\hline
\end{tabular}

Terms of seventh order.

\begin{tabular}{|c|c|c|c|c|c|c|c|c|}
\hline 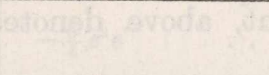 & 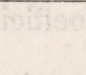 & 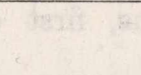 & 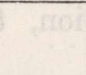 & & $L^{\prime}-\Theta^{\prime}$ & $L^{\prime}-\Pi^{\prime}$ & $L-\Theta$ & $L-\Pi$ \\
\hline$(450)^{i}\left(\frac{1}{2} e\right)^{7}$ & $(450$ & .. 450$)$ & . & $\cos$ & $i$ & 0 & $-i$ & +7 \\
\hline$(451)^{i}\left(\frac{1}{2} e\right)^{6}\left(\frac{1}{2} e^{\prime}\right)^{2}$ & $(451$ & .. 451) & . & $"$ & $i$ & 1 & $-i$ & +6 \\
\hline$(452)^{i}\left(\frac{1}{2} e\right)^{5}\left(\frac{1}{2} e^{\prime}\right)^{2}$ & $(452$ & . 452$)$ & $\ldots$ & , & $i$ & 2 & $-i$ & +5 \\
\hline$(453)^{i}\left(\frac{1}{2} e\right)^{4}\left(\frac{1}{2} e^{\prime}\right)^{3}$ & $(453$ & .. 453) & & " & $i$ & 3 & $-i$ & +4 \\
\hline$(454)^{i}\left(\frac{1}{2} e\right)^{3}\left(\frac{1}{2} e^{\prime}\right)^{4}$ & $(454$ & .. 454) & $\ldots$ & " & $i$ & 4 & $-i$ & +3 \\
\hline$(455)^{i}\left(\frac{1}{2} e\right)^{2}\left(\frac{1}{2} e^{\prime}\right)^{5}$ & $(455$ & .. 455$)$ & . & " & $i$ & 5 & $-i$ & +2 \\
\hline$(456)^{i}\left(\frac{1}{2} e\right)\left(\frac{1}{2} e^{\prime}\right)^{6}$ & $(456$ & . 456$)$ & & " & $i$ & 6 & $-i$ & +1 \\
\hline$(457)^{i}\left(\frac{1}{2} e^{\prime}\right)^{7}$ & $(457$ & $457)$ & $\ldots$ & , & $i$ & 7 & $-i$ & 0 \\
\hline$(458)^{i}\left(\frac{1}{2} e\right)^{5} \eta^{2}$ & $(458$ & $458)$ & & , & $i$ & 0 & $-i+2$ & +5 \\
\hline
\end{tabular}


Terms of seventh order (concluded):

\begin{tabular}{|c|c|c|c|c|c|c|c|}
\hline & & & & $L^{\prime}-\Theta^{\prime}$ & $L^{\prime}-\Pi^{\prime}$ & $L-\Theta$ & $L-\Pi$ \\
\hline$(459)^{i}\left(\frac{1}{2} e\right)^{4}\left(\frac{1}{2} e^{\prime}\right) \eta^{2}(459$ & . . 459) & & $\cos$ & $i$ & +1 & $-i+2$ & +4 \\
\hline$(460)^{i}\left(\frac{1}{2} e\right)^{3}\left(\frac{1}{2} e^{\prime}\right)^{2} \eta^{2}(460$ & . 460$)$ & $\cdots$ & $"$ & $i$ & +2 & $-i+2$ & +3 \\
\hline$(461)^{i}\left(\frac{1}{2} e\right)^{2}\left(\frac{1}{2} e^{\prime}\right)^{3} \eta^{2}(461$ & .. 461) & $\cdots$ & $”$ & $i$ & +3 & $-i+2$ & +2 \\
\hline$(462)^{i}\left(\frac{1}{2} e\right)\left(\frac{1}{2} e^{\prime}\right)^{4} \eta^{2}(462$ & .. 462) & . & $"$ & $i$ & +4 & $-i+2$ & +1 \\
\hline$(463)^{i}\left(\frac{1}{2} e^{\prime}\right)^{5} \eta^{2}$ & .. 463) & . & , & $i$ & +5 & $-i+2$ & 0 \\
\hline$(464)^{i}\left(\frac{1}{2} e\right)^{3} \eta^{4}$ & . 464$)$ & . & ” & $i$ & 0 & $-i+4$ & +3 \\
\hline$(465)^{i}\left(\frac{1}{2} e\right)^{2}\left(\frac{1}{2} e^{\prime}\right) \eta^{4}(465$ & .. 465) & & ” & $i$ & +1 & $-i+4$ & +2 \\
\hline$(466)^{i}\left(\frac{1}{2} e\right)\left(\frac{1}{2} e^{\prime}\right)^{2} \eta^{4}(466$ & . 466$)$ & $\cdots$ & $"$ & $i$ & +2 & $-i+4$ & +1 \\
\hline$(467)^{i}\left(\frac{1}{2} e^{\prime}\right)^{3} \eta^{4}$ & .. 467) & . & $"$ & $i$ & +3 & $-i+4$ & 0 \\
\hline$(468)^{i}\left(\frac{1}{2} e\right) \eta^{6}$ & . . 468) & & $"$ & $i$ & 0 & $-i+6$ & +1 \\
\hline$(469)^{i}\left(\frac{1}{2} e^{\prime}\right) \eta^{6}$ & . . 469) & & $"$ & $i$ & +1 & $-i+6$ & 0 \\
\hline
\end{tabular}

Here the several coefficients are $\mathrm{u}^{1}$ timately given in terms of the before-mentioned quantities $A^{i}, B^{i}, C^{i}, D^{i}, E^{i}, G^{i}, H^{i}, L^{i}, S^{i}, T^{i}$ (functions of $a, a^{\prime}$ ), and their differential coefficients in regard to $a$

$$
\left(A_{1}^{i}=\frac{1}{1} a \frac{d}{d a} A^{i}, \quad A_{2}^{i}=\frac{1}{1.2} a \frac{d^{2}}{d a^{2}} A^{i}, \& c .\right)
$$

as follows:-we have Leverrier, pp. 299-330, a list of functions (1), (2), .. (154) of the form $(1)=\frac{1}{2} K^{i},(2)=-2 h^{2} K^{i}+K_{1}^{i}+K_{2}^{i},(3)=-2 i^{2} K^{i}+K_{1}^{i}+K_{2}^{i}$, \&c., involving $i, h$, and $K^{i}$ and its derived functions $K_{1}^{i}, K_{2}^{i}$, \&c. The coefficients of the several cosines are given by means of the functions in question, thus, first coefficient, above denoted as $(1)^{i}(1 \ldots 20)$, is

$$
=(1)^{i}+(2)^{i}\left(\frac{1}{2} e\right)+(3)^{i}\left(\frac{1}{2} e^{\prime}\right) \ldots+(20)^{i} \eta^{6}
$$

where $(1)^{i}=(1),(2)^{i}=(2) \ldots$ writing in the functions (1), (2) $\ldots(10), h=i$, and $K^{i}=A^{i}$;

$$
\begin{aligned}
& (11)^{i}=(1),(12)^{i}=(2) \text {, \&c., writing } h=i \text { and } K^{i}=-E^{i} \text {, } \\
& (20)^{i}=(1), \quad \text { writing } h=i \text { and } K^{i}=-H^{i} \text {, }
\end{aligned}
$$

and so on for the various component coefficients $(1)^{i},(2)^{i} \ldots(469)^{i}$.

But the resulting expressions, for the several integer values $i=-10$ to +10 , are worked out in the Addition II. (Numerical Tables for the Calculation of the Coefficients of the Development of the Disturbing Function), pp. 358-383. And this Addition contains also, indicated by the letters $\delta$ and $\Delta$ respectively, the expressions of the 
terms which experience an alteration in passing from the development of the reciprocal of the distance to those of the disturbing functions $m^{\prime}$ upon $m$, and $m$ upon $m^{\prime}$ respectively.

We have

Disturbing Function $m^{\prime}$ upon $m$

$$
=m^{\prime}\left\{-\frac{r \cos H}{r^{\prime 2}}+\frac{1}{\rho}\right\} \text {. }
$$

Disturbing Function $m$ upon $m^{\prime}$

$$
=m\left\{-\frac{r^{\prime} \cos H}{r^{2}}+\frac{1}{\rho}\right\} .
$$

The expressions of $-\frac{r \cos H}{r^{\prime}}$ and $-\frac{r^{\prime} \cos H}{r^{2}}$, developed to the third order in the eccentricities and inclination, are given, Leverrier, pp. 272 and 274. Expressed in the terms of the foregoing arguments $L^{\prime}-\Theta^{\prime}$, \&c., and in terms of $a, a^{\prime}$ in place of $a$

\begin{tabular}{|c|c|c|c|c|c|c|}
\hline$-\frac{r \cos H}{r^{\prime 2}}=\frac{a}{a^{\prime 2}}$ into & & & $L^{\prime}-\Theta^{\prime}$ & $L^{\prime}-\Pi^{\prime}$ & $L-\Theta$ & $L-\Pi$ \\
\hline$-1+\frac{1}{2}\left(e^{2}+e^{\prime 2}\right)+\eta^{2}$ & . & $\cos$ & 1 & 0 & -1 & 0 \\
\hline $\begin{array}{lll}-e e^{\prime} & \ldots & \ldots\end{array}$ & $\cdots$ & $"$ & +1 & +1 & -1 & -1 \\
\hline$+\frac{3}{2} e-\frac{3}{4} e e^{\prime 2}-\frac{3}{2} e \eta^{2}$ & . & $"$ & +1 & 0 & -1 & +1 \\
\hline$-\frac{1}{2} e+\frac{1}{4} e e^{\prime 2}+\frac{3}{8} e^{3}+\frac{1}{2} e \eta^{2}$ & . & ” & +1 & 0 & -1 & -1 \\
\hline$-2 e^{\prime}+e^{2} e^{\prime}+\frac{3}{2} e^{\prime 3}+2 e^{\prime} \eta^{2}$ & . & " & +1 & +1 & -1 & 0 \\
\hline $\begin{array}{lll}-\frac{3}{4} e^{2} e^{\prime} & \ldots & \ldots\end{array}$ & . & , & +1 & +1 & -1 & -2 \\
\hline$+\frac{3}{16} e e^{\prime 2}$ & . & " & -1 & +2 & +1 & -1 \\
\hline$\ldots$ & . & " & +1 & +2 & -1 & -1 \\
\hline $\begin{array}{llll}+\frac{3}{2} e \eta^{2} & \ldots & \ldots & \ldots\end{array}$ & $\cdots$ & " & +1 & 0 & +1 & -1 \\
\hline
\end{tabular}
and $\alpha$, these are as follows: 


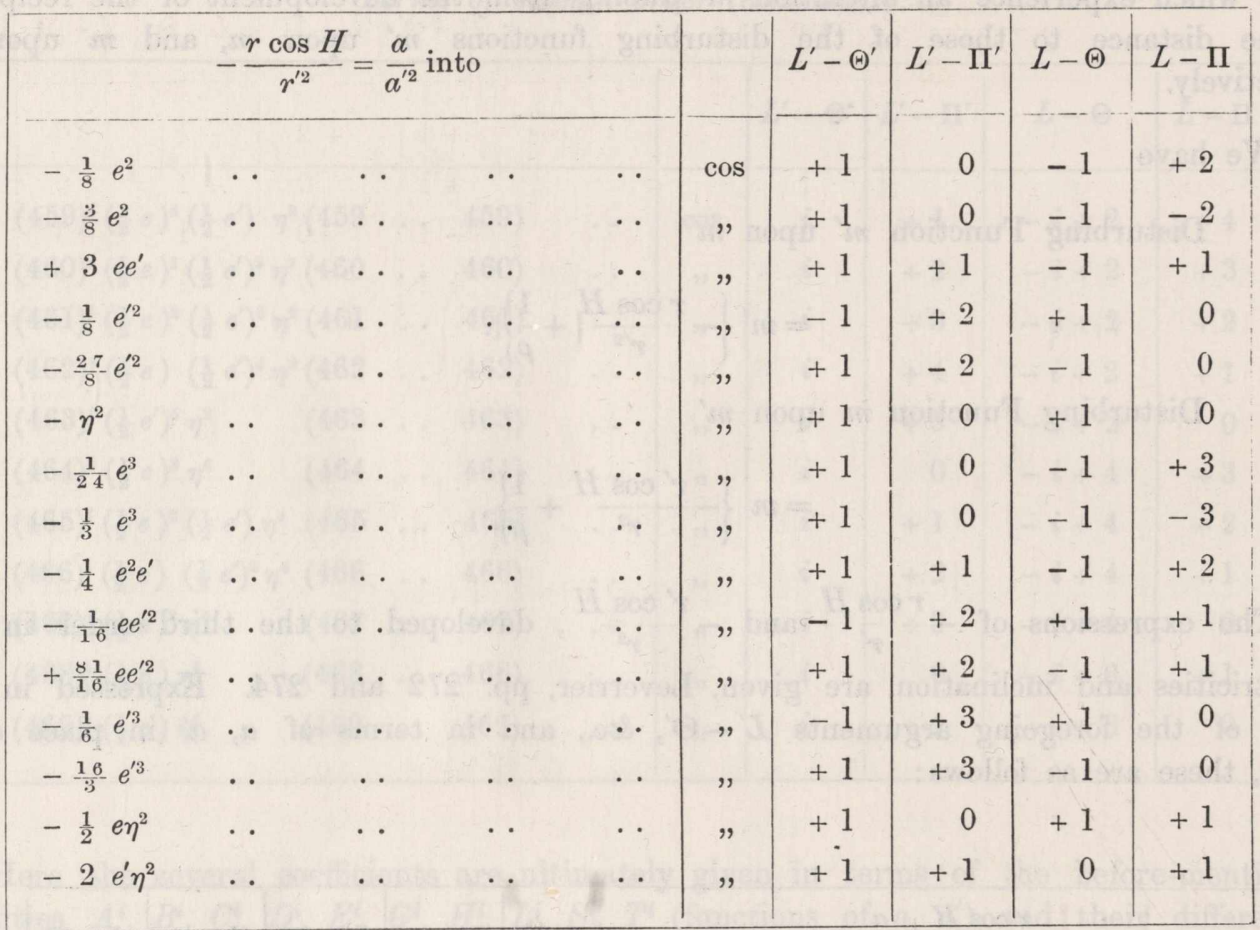

\begin{tabular}{|c|c|c|c|c|c|c|c|}
\hline$-\frac{r^{\prime} \cos H}{r^{2}}=\frac{a^{\prime}}{a^{2}}$ into & & & - & $L^{\prime}-\Theta^{\prime}$ & $L^{\prime}-\Pi^{\prime}$ & $L-\Theta$ & $L-\Pi$ \\
\hline$-1+\frac{1}{2}\left(e^{2}+e^{\prime 2}\right)+\eta^{2}$ & & . & $\cos$ & 1 & 0 & -1 & 0 \\
\hline $\begin{array}{llll}-e e^{\prime} \ldots & \ldots & \ldots\end{array}$ & . & $\cdots$ & ", & +1 & +1 & -1 & -1 \\
\hline$-2 e+e e^{\prime 2}+\frac{3}{2} e^{3}+2 e \eta^{2}$ & . & $\cdots$ & $"$ & +1 & 0 & -1 & -1 \\
\hline$+\frac{3}{2} e^{\prime}-\frac{3}{4} e^{2} e^{\prime}-\frac{3}{2} e^{\prime} \eta^{2} \ldots$ & & $\cdots$ & " & -1 & +1 & +1 & 0 \\
\hline$-\frac{1}{2} e^{\prime}+\frac{1}{4} e^{2} e^{\prime}+\frac{3}{8} e^{\prime 3}+\frac{1}{2} e^{\prime} \eta^{2}$ & . & $\cdots$ & , & +1 & +1 & -1 & 0 \\
\hline$+\frac{3}{16} e^{2} e^{\prime} \quad \ldots \quad \quad \ldots$ & . & $\cdots$ & $”$ & +2 & -1 & -2 & +2 \\
\hline$-\frac{2}{16} \frac{7}{6} e^{2} e^{\prime}$ & . & $\cdots$ & ” & +1 & +1 & -1 & -2 \\
\hline$-\frac{3}{4} e e^{\prime 2}$ & . & $\cdots$ & " & +1 & +2 & -1 & -1 \\
\hline$+\frac{3}{2} e^{\prime} \eta^{2}$ & . & $\cdots$ & $"$ & +1 & -1 & +1 & 0 \\
\hline$-\frac{1}{8} e^{2}$ & . & $\cdots$ & " & +1 & 0 & -1 & +2 \\
\hline$-\frac{27}{8} e^{2}$ & . & . & $"$ & +1 & 0 & -1 & -2 \\
\hline$+3 e e^{\prime}$ & . & . & $"$ & -1 & +1 & +1 & +1 \\
\hline$-\frac{1}{8} e^{\prime 2}$ & . & $\cdots$ & " & -1 & +2 & +1 & 0 \\
\hline
\end{tabular}




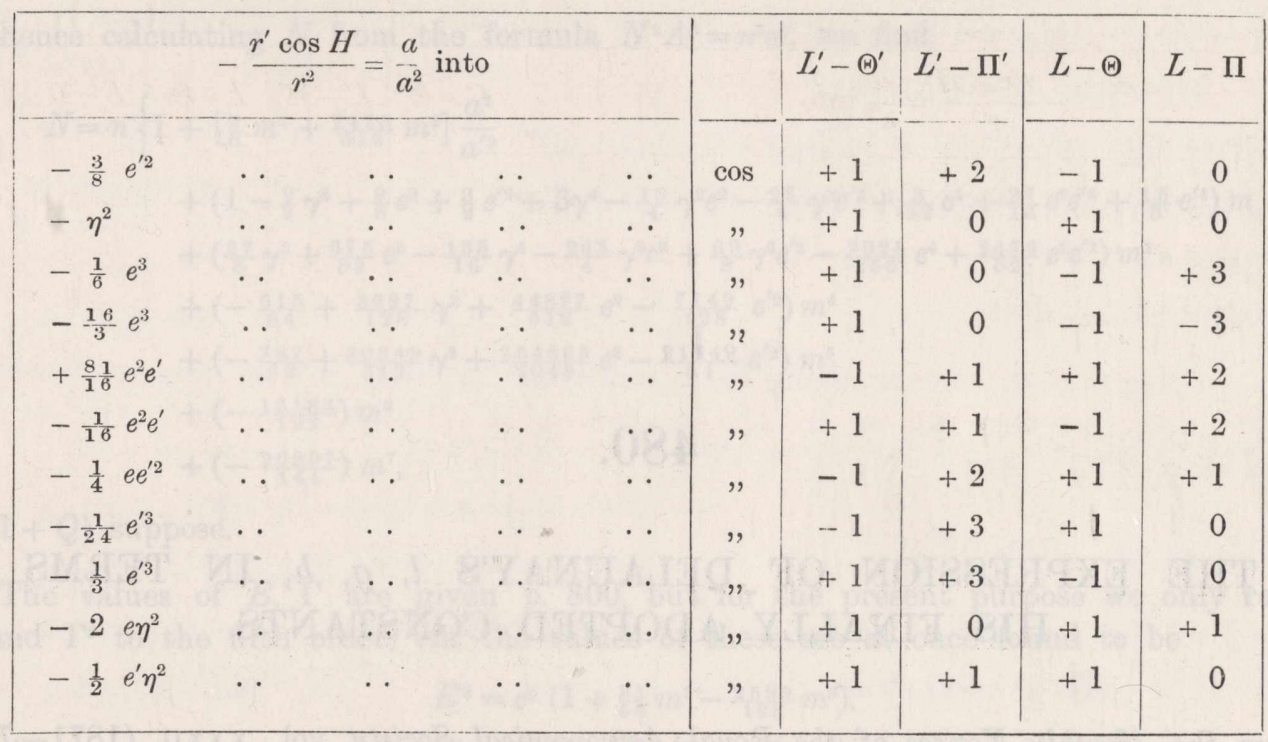

It is hardly necessary to observe that, to obtain the expressions of the Disturbing Functions, these additional terms are to be combined with the corresponding terms in the expression of the reciprocal of the distance: thus, in the Disturbing Function $\Omega\left(m^{\prime}\right.$ upon $\left.m\right)$, the entire term depending on $\cos \left[L^{\prime}-\Theta^{\prime}-(L-\Theta)\right]$ is

$$
=m^{\prime}\left\{2(1, \ldots 20)_{i=1}+\frac{a}{a^{\prime 2}}\left(-1+\frac{1}{2}\left(e^{2}+e^{\prime 2}\right)+\eta^{2}\right)\right\} \cos \left[\left(L^{\prime}-\Theta^{\prime}\right)-(L-\Theta)\right],
$$

where, however, the supplemental term is taken to the third order only. 